\title{
A Modification on Hamilton-Jacobi (HJ) Equation for Computing Wall Distances
}

\author{
By Tameo NAKANISHI \\ Department of Mechanical Systems Engineering, Yamagata University, Yonezawa, Japan
}

(Received May 23rd, 2005)

Key Words: CFD, Turbulence Model, Wall Distance

\section{Introduction}

The distance to the wall boundary, $d$, is required in many turbulence models. ${ }^{1-3)}$ Computing the wall distance for engineering flow problems of complex geometry is still a very difficult task. The conventional method of directly computing the geometrical distance is expensive, especially for moving body problems. ${ }^{4)}$ Difficulties for multiple wall problems may be encountered in some situations. Continuous efforts have been made to reduce the cost of computing wall distances. ${ }^{4-6)}$

For practical purposes, accurate wall distance is only required close to walls for turbulence model applications. Several approaches for solving differential equations ${ }^{5,6)}$ have been proposed for this purpose in the last decade. These approaches are more efficient and flexible as compared to geometrical methods. The latest one ${ }^{6)}$ is to solve the Hamilton-Jacobi (HJ) equation of $\phi$ :

$$
\nabla \phi \cdot \nabla \phi=1+\varepsilon \phi \nabla^{2} \phi
$$

where $\varepsilon$ is a positive constant. For a convex wall of negligible curvature, the solution of $\phi$ is a good approximation of $d$ in the very-near-wall region, $\phi$ becomes slightly larger than $d$ beyond this region and $\phi<d$ in the far-field region. The $\mathrm{HJ}$ equation may be viewed as an advection-diffusion equation and solved by the conventional upwind method. ${ }^{6}$ ) This approach, however, have several drawbacks.

1) For a convex wall having large curvature, which is the case of the leading and the trailing edges of an airfoil, $\nabla^{2} \phi \gg 0$, thus $|\nabla \phi|=\sqrt{1+\varepsilon \phi \nabla^{2} \phi}>1$. $\phi$ quickly deviates from $d$, resulting in $\phi>d$ away from the wall. The solution of $\phi$ cannot be considered as the approximation of $d$ where the wall distance is still important for turbulence modeling. Reducing the magnitude of $\varepsilon$ may improve the accuracy but increase numerical instability.

2) In a turbulence flow computation, an extremely fine grid $\left(O\left(10^{-6}\right)\right)$ with a dimensionless length for a Reynolds number of several million is employed. The pseudo-time step length for an unwind scheme may be restricted as the same order of the smallest grid space. Numerous iterations have to be conducted to reach a steady solution.

3) An upwind scheme is more complicated to code and

(C) 2006 The Japan Society for Aeronautical and Space Sciences more expensive than a central difference scheme, especially for an unstructured grid.

In the present paper, a modification is made in the $\mathrm{HJ}$ equation to eliminate the influence of the convex wall curvature so that the solution of $\phi$ maintains uniform approximation for the wall distance up to the mid-near-wall region. A non-linear numerical viscosity is introduced to stabilize the central difference solution of the HJ equation. These improvements enable simple and accurate computation of wall distances.

\section{Modification on HJ Equation}

The constant $(\varepsilon)$ in the HJ equation (1) is given by unity throughout the present paper. The effect of the wall curvature may be illustrated by considering the solution of $\phi$ outside of a two-dimensional (2D) cylinder with radius $R$. In this case, the Laplacian is written as

$$
\nabla^{2} \phi=\frac{1}{R+r} \frac{\partial \phi}{\partial r}+\frac{\partial^{2} \phi}{\partial r^{2}},
$$

where $r$ is the distance from the cylinder surface (independent variable). Equation (1) is simplified as

$$
\left(\frac{\partial \phi}{\partial r}\right)^{2}=\left(1+\phi \frac{\partial^{2} \phi}{\partial r^{2}}\right)+\frac{\phi}{R+r} \frac{\partial \phi}{\partial r} .
$$

The second term in the right-hand-side (i.e., denoted by $C V$ ) is due to the wall curvature and is positive for a convex wall. As $R \rightarrow 0$, the $C V$ term becomes extremely large, which can cause $(\partial \phi / \partial r)^{2}$ (the left-hand-side of the equation) to be much greater than unity in the mid-near-wall region. The effect of the wall curvature can simply be excluded by removing the $C V$ term in the $2 \mathrm{D}$ cylinder case. In a general problem, eliminating the curvature effect requires an approximation of this term. We approximate the curvature $(\kappa)$ by

$$
\kappa=\nabla \cdot \boldsymbol{n}, \boldsymbol{n}=\frac{\nabla \phi}{|\nabla \phi|+\varepsilon_{0}},
$$

where $\boldsymbol{n}$ is the unit normal vector and $\varepsilon_{0}=10^{-12}$ is a small constant added to prevent zero division. The $C V$ term is expressed as

$$
C V=\phi(\nabla \cdot \boldsymbol{n})|\nabla \phi| .
$$

Subtracting this term from the Laplacian can eliminate 
the effect of the wall curvature. The HJ equation (1) is therefore modified as follows:

$$
|\nabla \phi|^{2}=1+\phi\left\{\nabla^{2} \phi-\max (0,|\nabla \phi| \nabla \cdot \boldsymbol{n})\right\},
$$

where the max function is devised to retain the preferred curvature effect in the case of a concave boundary $(\nabla \cdot \boldsymbol{n}<0)$.

A stable solution for the central difference approximation of Eq. (6) can be obtained by introducing the nonlinear numerical viscosity $(v)$ of the following:

$$
\begin{gathered}
|\nabla \phi|^{2}=1+(\phi+v)\left\{\nabla^{2} \phi-\max (0,|\nabla \phi| \nabla \cdot \boldsymbol{n})\right\} \\
v=0.001(1-|\nabla \phi|)^{2} .
\end{gathered}
$$

Obviously, the numerical viscosity has almost no influence on the converged solution in the region where $|\nabla \phi| \rightarrow$ 1. Any existing iterative method is applicable to solve Eq. (7). In the present paper, the SOR method is employed.

\section{Numerical Examples}

We provide three numerical examples of using curvilinear coordinate systems. The following wall shapes along with the circular outer boundary of radius $R_{\text {out }}$ are considered: (a) cylindrical cylinder of different radiuses, (b) ellipse of an aspect ratio of 100, and (c) a 10-petal flower. All of these geometries are specified by the following formulation with different parameters:

$$
\begin{aligned}
& x=(R+H \cos n \theta) \cos \theta \\
& y=(R \cdot A+H \cos n \theta) \sin \theta .
\end{aligned}
$$

The curvilinear meshes are easily generated by linear interpolation of the wall nodes and the outer boundary nodes. No effort has been made to improve the quality of the mesh. The outer boundary is either considered as a far-field boundary or a wall boundary.

\subsection{Cylindrical cylinder of different radiuses}

This case is conducted to describe the influence of wall curvature. The parameters are given by: $A=1, H=0$, $R_{\text {out }}=10$ and (a) $R=0.005$, and (b) $R=0.5$. An O-grid of $201 \times 81$ grid points is employed where the minimum grid space is $10^{-6}$. The over-relaxation factor of the SOR method was given by $\omega=1.6$. Applying the central difference approximations along with non-linear numerical viscosity, converged solutions are obtained for both of the standard and modified $\mathrm{HJ}$ equations. The $L_{2}$ residual reaches the machine error of $10^{-16}$ at the iteration number of 2500 for the modified HJ equation. Figure 1 shows the computed $\phi$ outside the cylindrical cylinders. The $\phi$ obtained by the standard HJ equation (Fig. 1(a)) is much greater than $r$ throughout almost the entire region except for $r<0.002$ (not visible) and the far-field region $r>7.6$. Consequently, $\phi$ cannot be used as an approximation of the wall distance. In comparison, $\phi$ computed from the modified HJ equation (Fig. 1(b)) exhibits accurate approximation of the wall distance up to $r=2.5$. The solutions of $\phi$ for different radiuses fall on the same line. The error of the computed wall distance $\phi$ is within $0.37 \%$ in the region $r \leq 0.87$.

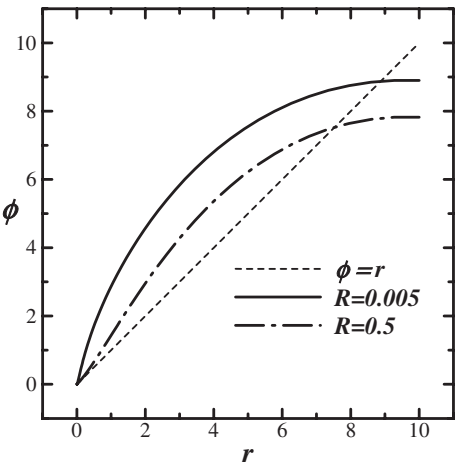

(a) Standard HJ equation

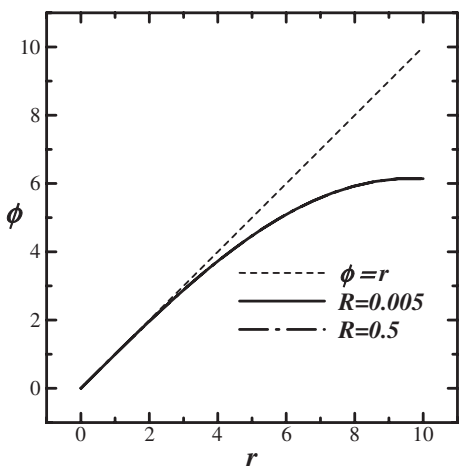

(b) Modified HJ equation

Fig. 1. Computed $\phi$ outside the cylindrical cylinders of different radiuses.

\subsection{Ellipse of an aspect ratio of $\mathbf{1 0 0}$}

The parameters are given by $A=0.01, H=0, R=0.5$ and $R_{\text {out }}=10$. The O-grid of $201 \times 81$ grid points was employed where the minimum grid space is $10^{-6}$. Figure 2 shows (a) meshes and (b) contours of $\phi$ in the near-wall region. Given $\omega=1.6$, a fully converged solution is achieved at the iteration number of 3000 . Accurate wall distances were obtained in terms of $\phi$ in spite of the use of highly inclined meshes.

\subsection{A ten petal flower}

The parameters are given by $A=1, H=0.8, R=1, n=$ 10 and $R_{\text {out }}=2$. In this case, both the flower surface and the outer boundary are considered as body surfaces to simulate the situation of an internal flow. The O-grid of $401 \times 81$ grid points was employed where the minimum grid space next to the body surfaces is $1.4 \times 10^{-6}$. Figure 3 shows (a) part of meshes, and (b) contours of $\phi$. Desirable distributions of $\phi$ as the approximation of the wall distances were obtained again in spite of the use of highly inclined/screwed meshes. For this case, an under relaxation factor of $\omega=0.6$ for the SOR method was employed to achieve convergence. Fully converged solution was obtained at the iteration number of 2000 .

\section{Conclusions}

A modification on the $\mathrm{HJ}$ equation for computing wall distances has been conducted to exclude the influence of the wall curvature. A non-linear numerical viscosity has 


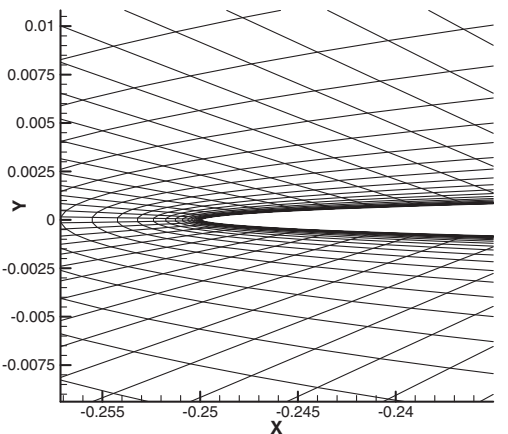

(a) Meshes

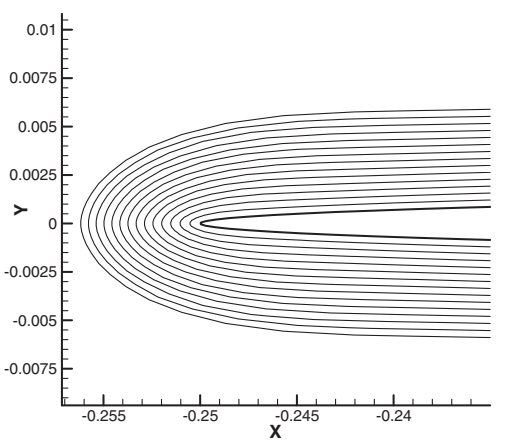

(b) Contours of $\phi(0 \leq \phi \leq 0.005,15$ divisions $)$ in the near-wall region

Fig. 2. Computed $\phi$ of an ellipse of an aspect ratio of 100 .

been proposed to stabilize the central difference solution of the modified HJ equation. The solution of the modified HJ equation achieves an accurate approximation of the wall distance up to the mid-near-wall region. The numerical scheme is inexpensive, straightforward and can be incorporated into any turbulence model.

\section{References}

1) Baldwin, B. S. and Lomax, H.: Thin Layer Approximation and Algebraic Model for Separated Turbulent Flows, AIAA Paper 78-257, 1978.

2) Baldwin, B. S. and Barth, T. J.: A One-Equation Turbulence Transport

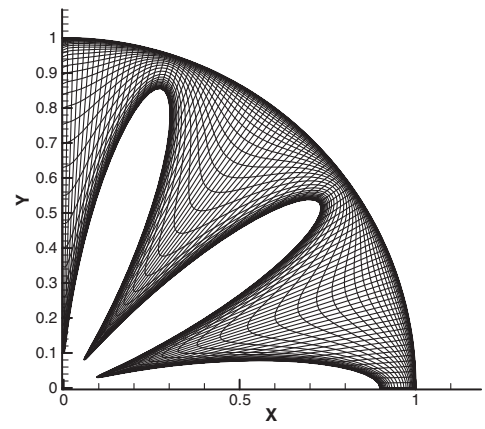

(a)

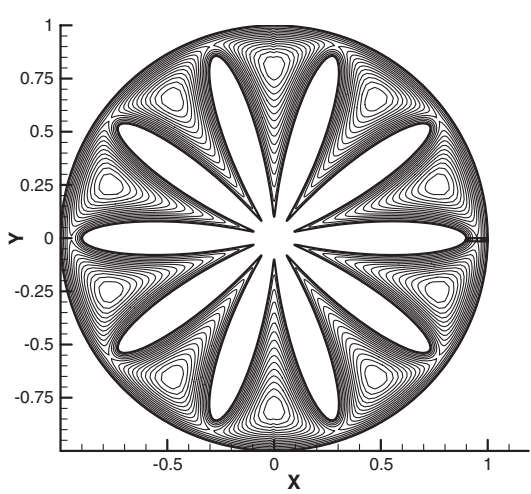

(b)

Fig. 3. Part of meshes (a), and contours of $\phi$ for complicated internal flow situation (b).

Model for High Reynolds Number Wall-Bounded Flows, AIAA Paper 91-0610, 1991.

3) Spalart, P. R. and Allmaras, S. R.: A One-Equation Turbulence Model for Aerodynamic Flows, La Recherche Aerospatiale, 1 (1994), pp. 5-21.

4) Boger, D. A.: Efficient Method for Calculating Wall Proximity, AIAA J., 39 (2001), pp. 2404-2406.

5) Osher, S. and Sethain, J. A.: Fronts Propagating with Curvature Dependent Speed: Algorithms based on Hamilton-Jacobi Formulation, J. Comput. Phys., 79 (1988), pp. 12-49.

6) Tucker, P. G.: Differential Equation-Based Wall Distance Computation for DES and RANS, J. Comput. Phys., 190 (2003), pp. 229-248. 